LIAMES 1 - pp. 105-123, Primavera 2001

\author{
J. Pedro Viegas Barros \\ (CONICET-Universidad de Buenos Aires)
}

\title{
Evidencias de la Relación Genética Lule-Vilela
}

\begin{abstract}
:
This article presents the existing evidence for a Lule-Vilela genetic relationship. Lule and Vilela are probably the two worst known languages from the Chaquean region, and is no consensus yet about their classification.

The phonological systems (the Lule one being a temptative phonemization) of both languages are discussed. The, the phonological correspondences are showed, and later the grammatical and lexical cognate sets. No attempt is made for reconstructing the Proto-Lule Vilela phonological system.
\end{abstract}

\section{INTRODUCCIÓN. LAS LENGUAS LULE Y VILELA Y LA CUESTIÓN DE SU CLASIFICACIÓN.}

El presente es un trabajo comparativo entre el lule y el vilela, dos de las lenguas peor conocidas de la región chaqueña austral*. El lule se habló en parte de las actuales provincias argentinas de Tucumán y Santiago del Estero, al oeste del río Salado, llegando por el norte hasta el sur del valle del río Sali, en Jujuy. Extinguido posiblemente desde fines del siglo XVIII, es conocido principalmente gracias al pequeño Arte, vocabulario y textos religiosos, publicados por Antonio Machoni en 1732. Las demás fuentes son el vocabulario, mucho menor, del abate Ferragut, publicado por Felipe S. Gilij en 1782, y palabras y frases sueltas, así como un par de oraciones cristianas, dispersas en la obra de otros escritores jesuitas.

* Agradezco las valiosas observaciones bibliográficas realizadas por el profesor Alain Fabre, de la Universidad Tecnológica de Tampere, Finlandia. Asimismo manifiesto aquí mi más profundo reconocimiento a Diana Balmori hija de Clemente Hernando Balmori, quien tuvo la gentileza de remitirme desde los Estados Unidos copia de una libreta de campo con importante material inédito vilela compilado por su padre en el Chaco durante la década de 1960 
El vilela se habló entre los ríos Bermejo y Salado, en una zona que incluía el norte de la actual provincia de Chaco, parte del oeste de Formosa y algo del sudeste de la de Salta, y áreas fronterizas de Santiago del Estero. En los 60, dos lingüistas, Hernando Balmori y Lozano, trabajaron con quienes parecen haber sido los últimos hablantes fluidos. Recientemente (Terán 1995) se ha reportado la presencia de personas que todavía recuerdan por lo menos algunas palabras. Adelaar (1991: 56) cita al vilela como una de las lenguas aborígenes argentinas que requieren investigación urgente.

Aunque desde fines del siglo pasado, ambas lenguas han sido generalmente clasificadas en una misma familia lingüística, hasta el momento no se ha publicado un trabajo de comparación extenso entre ellas, dentro del marco teórico y metodológico de la lingüística histórico-comparativa. A raíz de ello, muchos investigadores pusieron en duda, o incluso negaron, el parentesco entre ambas lenguas. Así, p. ej., Loukotka (1968: 55, 277) las clasificó en grupos distintos. Tovar tampoco aceptó su parentesco, basándose en que, según su propia investigación, su léxico (Tovar y Larrucea de Tovar 1984: 35) “...acusa escasas semejanzas". Kaufman se limita a considerar (1990: 46) "buena" la hipótesis de relación, sin admitir que la misma esté probada. Y Fabre, por su parte, sólo dice (1998: 1192) que ambas lenguas “...según parece...” formarían una familia.

En este trabajo se revisan las evidencias de parentesco entre ambas lenguas presentadas por diversos autores, y se proponen otras nuevas. En las conclusiones, se examinan los resultados, que muestran la existencia de un vínculo genealógico Lule-Vilela.

\section{SISTEMAS FONOLÓGÍCOS}

\subsection{Lule}

El sistema fonológico mínimo que se puede deducir para la lengua lule, a partir de las fuentes disponibles, es el siguiente:

Consonantes:

$\begin{array}{lcccccc} & \text { Labial } & \text { Dental } & \text { Alveolar } & \text { Palatal } & \text { Velar } & \text { Glotal } \\ \text { Nasales } & \mathbf{m} & \mathbf{n} & & & & \\ \text { Plosivas Simples } & \mathbf{p} & \mathbf{t} & \mathbf{c} & & \mathbf{k} & \mathbf{P} \\ \text { Plosivas Glotal. } & \mathbf{p} & \mathbf{t} & \mathbf{c} & & \mathbf{k} \text { ' } & \\ \text { Fricativas } & & \mathbf{t} & \mathbf{s} & & & \\ \text { Aproximantes } & \mathbf{w} & \mathbf{l} & & \mathbf{y} & & \end{array}$

Vocales:

Altas

Medias

Baja

Anteriores
$\mathbf{i}$
$\mathbf{e}$

Central

Posteriores

u

o 
La fonemización de una parte de los grafemas utilizados en las fuentes no presenta mayores problemas. Interpreto las letras usadas por Machoni $\langle\mathbf{z}\rangle$ y $\langle\boldsymbol{c}\rangle, y\rangle \mathbf{c}\rangle$ ante vocal anterior, como representando una africada /c/; otras fuentes pueden emplear $\langle\mathbf{c h}\rangle$ en su lugar. No está claro qué representa la duplicación de consonantes. En algunos casos, la correspondencia con formas vilela parece indicar glotalizadas, en otros casos se trata con mayor probabilidad de grupos de dos consonantes iguales, surgidos por caída de una vocal de una sílaba reduplicada. Una excepción es el grupo <ll $>$, que la mayor parte de las veces parece representar, de acuerdo a los cognados vilela, una fricativa lateral sorda / $\mathbf{t} /$ : interpreto como representación de este fonema, además, a los dígrafos $\langle\mathbf{h l}\rangle,\langle\mathbf{h}\rangle\rangle \mathrm{y}\langle\mathbf{s} \mathbf{}\rangle$. Pero $\langle$ sl $>$ también puede representar el grupo consonántico /sl/, p. ej. en los casos en que se aplica el prefijo verbal $\boldsymbol{s}$ - a raíces que empiezan con $\boldsymbol{l}$-, como en slem- 'horadar', de lem-p 'agujero'. El grupo gráfico <tqu > representa seguramente una glotalizada $/ \boldsymbol{k}$ '/ en la serie cognada <itqueto > "horrorosa cosa", dada la correspondencia / $k$ '/ en el cognado vilela, cf. (132). En cuanto al grafema $\langle\mathbf{h}\rangle$, su uso cuando hay una articulación glotalizada en el cognado vilela correspondiente (p. ej. <poho-> 'encanecer', vilela p'o- 'blanco') parece indicar que podría tratarse más bien de una oclusiva [?] que de una aproximante glotal [h], lo que sería congruente con el hecho de que los grupos de dos vocales iguales alternan con grupos de dos vocales con $\langle\mathbf{h}\rangle$ intercalada $(\langle\mathbf{V h V}\rangle)$, lo que estaría indicando la presencia de la frecuente "vocal eco" en presencia de la oclusiva glotal. También apunta a la existencia de [?], más bien que a [h], la siguiente aclaración de Machoni (1877: 124), sub voce. Apuntalar: "Seç, Seé. (En este verbo se pronuncia la s como si fuera sola sin juntarse con la e; al contrario que en el verbo Seç, que significa llorar)"; es decir, $\boldsymbol{s} \boldsymbol{P e}$ 'apuntalar' frente a se- 'llorar'. 'Apuntalar' también aparece escrito en otra parte (Machoni 1877: 188, s. v. "Puntal”) como <S. hé, S. heé>. En Machoni, el dígrafo <ch $>$ representa muy probablemente $/ \boldsymbol{k}$ '/ en un caso como (Machoni 1877: 151) <lacochóop> 'desvergonzado', donde el final <-chóop> es una variante de un sufijo negativo/privativo que también aparece escrito <-cop>, <-cóp>; pero en otros casos parece indicar [tš], representando -posiblemente- una variante de /c/: cf. (Machoni 1877: 169) <chucátiç, chucátyy> 'jugar a los naipes' (préstamo del Quechua, cf. Quichua Santiagueño [Bravo 1975: 42] čoqa- 'Tirar, arrojar... Tirar las cartas...') y quizás también en la interjección (Machoni 1877: 106) <achá> "del que se queja de frío". La letra <r> ocurre muy raramente en formas patrimoniales lule, y la he interpretado como representando un fono vibrante [r], que sería un alófono esporádico del fonema $/ \mathbf{l} /$, dado que en los préstamos del castellano /r/ es reemplazado por lule ///, como en teleko 'trigo', talapa 'trapo', etc.

Ejemplos de la distribución opositiva de las consonantes:

$/ \mathbf{m} / \mathbf{x} / \mathbf{p} /, / \mathbf{w} /, / \mathbf{n} /$

$\begin{array}{lll}\text { man 'puerco montés' } & \text { pan } & \text { 'posesivo 3a. p. pl.' } \\ \text { mama 'carcoma, polilla' } & \text { wawa } & \text { 'tigre' } \\ \text { mene- 'traer' } & \text { nene- } & \text { 'venir a prisa' }\end{array}$

$/ \mathbf{n} / \mathrm{x} / \mathbf{t} /, / \mathbf{t} /, / \mathbf{l} /$

nikamayc- 'adornar con las manos' tikamayc- 'enmendar(se)'

nup 'otro'

nep- 'quitar'

tup 'su diente'

lep- 'entrar' 


\begin{tabular}{|c|c|c|}
\hline \multicolumn{3}{|l|}{108} \\
\hline \multicolumn{3}{|l|}{$/ \mathbf{p} / \mathbf{x} / \mathbf{t} /, / \mathbf{k} /, / \mathbf{p}\left({ }^{\prime}\right) /, / \mathbf{w} /$} \\
\hline pokop 'podrido, apolillado' & tokop & 'su cabeza' \\
\hline apo-' 'buscar' & $\operatorname{ap}\left({ }^{\prime}\right) \boldsymbol{o}-$ & 'sahumar' \\
\hline pis 'nervio' & kis & ‘уо' \\
\hline pele 'hombre' & wele & 'segundo' \\
\hline \multicolumn{3}{|l|}{$/ \mathbf{t} / \mathrm{x} / \mathbf{c} /, / \mathbf{k} /, / \mathbf{t}\left({ }^{\prime}\right) /, / \mathbf{t} /, / \mathbf{l} /$} \\
\hline ati 'maduro' & $a c i$ & 'esp. de avispa' \\
\hline ati- 'madurar' & aki- & 'secar' \\
\hline atolo 'lagarto' & $a t(') o$ & 'flor' \\
\hline tup 'lienzo tupido' & tup & 'su diente' \\
\hline ate 'mazamorra' & ale- & ‘arder’ \\
\hline \multicolumn{3}{|l|}{$/ \mathbf{c} / \mathrm{x} / \mathbf{k} /, / \mathbf{c}\left({ }^{\prime}\right) /, / \mathbf{s} /$} \\
\hline cey 'piojo del cuerpo' & key & ‘frío' \\
\hline aci 'esp. de avispa' & $a c(') i s a-$ & 'estornudar' \\
\hline cey 'piojo del cuerpo' & sey & ‘cóndor’ \\
\hline \multicolumn{3}{|l|}{$/ \mathbf{k} / \mathrm{x} / \mathbf{k}\left({ }^{\prime}\right) /, / \mathbf{P} /$} \\
\hline ikep 'mucho, muy' & ik(')eto & 'horrible' \\
\hline $\boldsymbol{k} \boldsymbol{a}$ 'boca' & $P a$ & 'tierra' \\
\hline \multicolumn{3}{|l|}{$/ \mathbf{t} / \mathrm{x} / \mathrm{s} /, / \mathbf{l} /$} \\
\hline łu 'diente' & su & 'hormiga' \\
\hline łemilas 'chinche del monte' & lemis- & 'tener dolor de muelas' \\
\hline \multicolumn{3}{|l|}{$/ \mathbf{y} / \mathbf{x} / \mathbf{w} /, / \mathbf{l} /, / \mathbf{s} /$} \\
\hline yat- 'perderse, desaparecer' & wat- & 'recoger fruta' \\
\hline yak - 'plantar, sembrar' & lak- & 'estar boca abajo' \\
\hline yo? 'calabaza larga' & so?- & ‘amarillo’ \\
\hline \multicolumn{3}{|l|}{ /2/ x /Ø/ } \\
\hline$P e$ 'estiércol' & $e$ & 'palo, madera, árbol' \\
\hline \multicolumn{3}{|c|}{ Ejemplos de la distribución opositiva de las vocales: } \\
\hline \multicolumn{3}{|l|}{$/ \mathbf{i} / \mathbf{x} / \mathbf{e} /, / \mathbf{u} /$} \\
\hline ini 'sol, día' & ine & 'teta' \\
\hline eni 'hermano mayor' & enu & ‘casa' \\
\hline \multicolumn{3}{|l|}{$/ \mathbf{e} / \mathbf{x} / \mathbf{a} /, / \mathbf{o} /$} \\
\hline yet- 'bailar, danzar' & yat- & 'perderse, desaparecer' \\
\hline es- 'soltar' & os- & 'doler’ \\
\hline \multicolumn{3}{|l|}{$/ \mathbf{a} / \mathbf{x} / \mathbf{o} /$} \\
\hline asto 'quebracho blanco' & osto & ‘ajî’ \\
\hline
\end{tabular}


Viegas Barros: Evidencias de la Relación GenÉtica Lule-Vilela

$/ \mathbf{o} / \mathrm{x} / \mathbf{u} /$

co 'cielo'

cu 'ojos, granos'

\subsection{Vilela}

Los fonemas de la lengua vilela son los siguientes.

Consonantes:

\begin{tabular}{|c|c|c|c|c|c|}
\hline Nasales & $\begin{array}{l}\text { Labial } \\
\mathbf{m}\end{array}$ & $\begin{array}{c}\text { Dental } \\
\mathbf{n}\end{array}$ & Alveo-Palatal & Velar & Posvelar \\
\hline Sonoras & $\mathbf{b}$ & $\mathbf{r}$ & & $\mathbf{g}$ & \\
\hline Plosivas Simples & $\mathbf{p}$ & $\mathbf{t}$ & $\breve{\mathbf{c}}$ & $\mathbf{k}$ & \\
\hline Plosivas Glotal. & p' & $\mathbf{t}^{\prime}$ & с̌' & k' & \\
\hline Fricativas & & $\mathbf{1}$ & $\mathbf{s}$ & & \\
\hline Aproximantes & $\mathbf{w}$ & 1 & $\mathbf{y}$ & & \\
\hline
\end{tabular}

Vocales:

$\begin{array}{ccc}\text { Anteriores } & \text { Central } & \text { Posteriores } \\ \mathbf{i} & \mathbf{u} \\ \mathbf{e} & & \mathbf{o}\end{array}$

Medias

$\mathbf{a}$

Los cuadros anteriores se basan en Lozano (1970a: 85; 1977: 93), con dos modificaciones. Por una parte, el fono oclusivo glotal [?], considerado fonema por Lozano, alterna con $\boldsymbol{\emptyset}$ en las posiciones inicial y final (inicialmente también con [h]), posiciones en las que indudablemente no tiene relevancia fonológica. Como en los demás casos se encuentra siempre en límite de unidades significativas, la primera de las cuales termina con vocal y la siguiente empieza con vocal (p. ej. [ma Payem] 'yacaré', de ma 'agua' y ayem 'iguana', cf. las series (50) y (70)), este fono en realidad no tiene estatus fonológico. Por otra parte, [d] se encuentra únicamente en posición inicial, donde se encuentra en libre variación con [r]; por tal razón, parece preferible considerarlo simplemente un alófono de $/ \mathbf{r} /$, y no un fonema distinto.

\section{CORRESPONDENCIAS FONOLÓGICAS}

A partir de las series cognadas establecidas hasta el momento, es posible deducir las siguientes correspondencias entre L(ule) y V(ilela), donde los números entre paréntesis se refieren a las series cognadas que ilustran cada correspondencia .

1. L p, Vh, Ø/\#_o: (7), (113), (140), (178).

2. L p, V p: (6), (7), (26), (27), (33), (41), (49), (62), (64), (74), (75), (80), (81), (88), (104), (114), (120), (141), (145), (147), (149), (153), (157), (170), (181), (194), (197), (198). 
3. L p, V q, k: (43), (98)?, (121), (150).

4. L p'), V p': (139), (179).

5. Lw, V b: (9), *(20), (21), (60), (66), *(85), (189).

6. Lw, Vw: (46), (65), (89), (90), (94), (105), (115), (125), (146), (147), (190), (191), (192).

7.Lw, Vh: (102), (193).

8. L n/i, e, a_\#, V m: (23), (70), (80).

9. L m, V m: (4), (5), (12), (14), (34), (50), (52), (69), (77), (78), (79), (87), (89), (91), (108), (136), (137), (140), (172), (185).

10. Lt $\mathbf{V}$ t: (8), (15), (18), (24), (28), (29), (40), (45), (111), (125), (127), (132), (143), (183), (185), (194).

11. L t $\left(^{\prime}\right), \mathrm{V} \mathbf{t}^{\prime}:(13)$.

12. L t'('), V r $(\sim 1):(84),(90),(151),(186),(187)$.

13. Ln, V n: (31), (32), (35), (36), (48), (55), (61), (62), (63), (95), (98), (102), (103), (112), (116), (121), (123), (130), (133), (143), *(149), (152), (153), (172), (173), (174), (175), (176), (182), (190).

14. L t, V ł: (45), (61), (93), (101), (109), (117).

15. L1, V I: (52), (87).

16. Ll, V ł: (69), (133), (160), (162).

17. Ll, V l: (3), (4), (17), (20), (22), (30), (39), (57), (60), (76), (83), (86), (89), (91), (100), (111), (114), (115), (126), (134), (135), (140), (145), (147), (153), (154), (156), (157), (170), (175), (176), (179), (183), (184).

18. Ll, V r: (56), (126), (129).

19. Lc, V s: (43), (71), (88), (122), (128), (161), (174), (175).

20. L c, V $\breve{c:}(92),(127),(163),(184)$.

21. Lc, V t: (1), (10), (56), (131), (148).

22. Lc, V k: (1), (56), (79), (129), (142), (161), (162), (166).

23. Le'('), V t': (159).

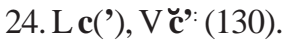

25. L s/_*i, V ł: (59), (127).

26. L s, V s: (2), (16), (25), (44), (57), (58), (79), (82), (83), (86), (96), (104), (105), (118), (124), (135), (138), (141), (142), (144), (159), (181), (188), (189), (193), (195), (200).

27. Ls, Vh: (37), (53), (64), (73), (103), (142), (165), (180), (182), (183), (191).

28. Ly, Vy: (19), (20), (38), (42), (54), (66), (67), (70), (91), (119), (120), (144), (165), (169), (194), (195), (196), (197), (198), (199).

29. L k, V k: (2), (11), (14), (15), (59), (60), (71), (73), (77), (87), (90), (98), (99), (105), (109), (115), (135), (147), (168), (170), (176).

30. L k, V q: (63), (68), (72), (74), (75), (76), (81), (110), (134), (169), (178).

31. L k('), V k': (38), (49), (97), (132), (171).

32.LP/V_V,Vh:(51), (196).

33.L 2, V Ø: (54), (67), (72), (83), (99), (105), (121), (128), (137), (139), (152), (153), (158), (167).

34. L Ø, V g: (86), (87)?, (108), (138), (145), (179), (188).

35. L Ø, V G: (122), (124).

36. L Ø, V h: (31), (74), (78), (100), (107), (119), (123), (136), (166).

37. L u, V u: (20), (43), (54), (77), (82), (86), (92), (93), (101), (105), (117), (118), (126), (151), (163), (175), (185), (199). 
38. Lu, V o: (76), (107), (120), (124), (140).

39. L u, V i: (42), (52), (88), (103), (106), (119), (123), (179), (182), (188), (200)?.

40. Lu, V e: (43), (111), (116), (140), (196).

41. L o/*q_p: V a: (74), (75).

42. Lo, V o: (4), (41), (49), (60), (63), (67), (68), (81), (90), (99), (136), (137), (139), (143), (144), (148), (176), (177), (178), (193).

43. Lo, V u: (44), (51), (135), (138), (150), (167), (194).

44. Li, V i: (2), (5), (11), (36), (48), (53), (59), (60), (64), (71), (80), (95), (96), (104), (109), (112), (127), (130), (131), (132), (133), (149), (159), (161), (162), (174), (176), (184), (187), (192), (195).

45. Li, V e: (11), (13), (16), (45), (47), (69), (124), (146), (170).

46. Le, V o/p_, w_,_w: (7), (94), (113), (146), (157).

47. Le, V e: (11), (13), (14), (15), (17), (22), (26), (27), (29), (33), (56), (95), (110), (122), (132), (153), (154), (160), (166), (173), (180), (183), (190), (198), (199).

48. Le, V i: (1), (15), (56), (57), (59), (108), (114), (129), (142), (154), (197).

49. Le, V a: (17), (39), (42), (50), (62), (94), (95), (100), (108), (117), (125), (131), (133), (165), (171), (191).

50. Le, Vwa: (116), (121), (155)?.

51. La, V o/q, k('): (68), (97), (134).

52. La, V e: (9), (29), (61), (65), (66), (70), (71), (83), (84), (89), (90), (91), (141), (148), (170), (186), (194).

53. La, V a: (9), (19), (46), (47), (55), (65), (66), (69), (70), (71), (74), (78), (79), (83), (89), (90), (91), (98), (102), (109), (115), (119), (126), (127), (128), (145), (152), (156), (159), (160), (168), (169), (195).

Parece que la vocal L e puede corresponder a las secuencias V aye, cf. (164) e iye, cf. (105), mientras que $\mathrm{L} \boldsymbol{i}$ puede corresponder a V eye , cf. (57) y a V ey, cf. (167).

$\mathrm{L} \boldsymbol{u}$ puede corresponder a la secuencias $\mathrm{V}(\boldsymbol{V}) \boldsymbol{b} \boldsymbol{V}$, cf. (20), (85), $\boldsymbol{w} \boldsymbol{V}$, cf. (158), e incluso $\boldsymbol{V g w} \boldsymbol{V}$, cf. (87).

\section{COGNADOS GRAMATICALES}

Utilizo las siguientes abreviaturas: Am = Ambrosetti (1894), AV = Adelung y Vater (1813), D = (Dobrizhoffer 1968), Fe = Ferragut (Gilij 1965 [1782]), Fo = Fontana (1881: 172), HB

= Hernando Balmori, HBR = Hernando Balmori en Rona (1964), He = Hervás (1800); J = Jolís (1972 [1789]), JG = Jolís en Gilij (1965 [1782]), Lo = Lozano, Ll = Llamas (1910), LN = Lehmann-Nitsche (1924-5), LQ = Lafone Quecedo, M = Machoni (1877 [1732]), MC = Martínez Crovetto, Pe = Pelleschi en LQ (1895).

\subsection{Pronombres y afijos personales}

(1) L (M) - $\boldsymbol{c}, \boldsymbol{- s}, \boldsymbol{- t}$ ' 1 a. p. sing. pos.'/'1a. p. sing. sujeto', $-\boldsymbol{c} \boldsymbol{e}-\boldsymbol{n}$ '1a. p. pl. pos.'; V (HB 1967, HBR, Lo 1964, 1970a) -ki -ti '1a. p. sing. sujeto'. 
(2) L (M) kis ‘yo'; V (AV, Lo 1967, 1970a) -ki-s ‘1a. p. pl. exclusivo sujeto', (AV, Am, HB 1967, HBR, Lo 1964, 1977) na-ki-s na-ti-s 'nosotros (exclusivo)'.

(3) L (M) $l \boldsymbol{e}$ - ; V (Lo 1970a, 1970b, 1977) $l$-, rup-l- '3a. p. objeto'.V rup- parece ser el elemento intensificador que aparece en (151).

(4) L (M) -lom '2a. p. pl. sujeto no imperativo/posesivo'; V (Lo 1964, HB 1967)-lem -lom '2a. p. pl. sujeto no imperativo', (HB 1967) -lom '2a. p. pl. imperativo', (Lo 1964, 1977) na-lem 'vosotros'.

(5) L (M) mi-l 'vosotros'; V(AV, HB 1967, BR, Lo 1964, 1970a) -m - mi '2a. p. sing. sujeto no imperativo/posesivo', (HB 1967) -o-m '2a. p. sing. sujeto imperativo', (AV, Pe, Am, Lo 1964, 1970a, 1977) na-m 'tú'.

(6) L (M) - $\boldsymbol{p}$ '3a. p. sing. sujeto no imperativo/posesivo', -pa-n '3a. p. pl. sujeto no imperativo/ posesivo’; V (HB 1967) - $\boldsymbol{p}$ ‘3a. p. sing. sujeto no imperativo', (HB 1967) -p - pa ‘3a. p. posesivo'.

(7) L (M) -pep; V (HB 1967) -op '3a. p. sing. sujeto imperativo'.

(8) L (M) -t '3a. p. sing. no imperativo', -to '3a. sing. imperativo'; V (HB 1967, HBR, Lo 1964, 1970a, 1977) -t $\sim$-te $\sim$-tek ‘3a. p. sing. y pl. no imperativo', (HB 1967) - $\boldsymbol{t}$ ‘3a. p. sing. posesivo'.

(9) L (M,AV) wa 'nosotros'; V(HB 1967, Lo 1964) -ba $\sim$-be $\sim$-ba-t $\sim$-be-t '1a. p. pl. inclusivo'.

\subsection{Demostrativos e interrogativos}

(10) L c - raíz interrogativa, cf. (11) y (M) c-ya ‘¿dónde?’, cf. (19); V t-ike $\sim$-ik ‘¿qué?’/ 'algo', t-ike-t ‘qqué?', cf. (11).

(11) L (M) c-ike- interrogativo (cike-p-ma ‘¿cuánto?', cike-to-le-ma ‘¿cuándo?', cike-p-le-ma ‘¿cuántas veces?', c-ike-ma '¿cuántos?'); V (Lo 1970a) eke, -ke marcador de oración interrogativa, (Ll, HB 1967, 1998, Lo 1970a) t-ike t-ik ‘¿qué?'/'algo', (Ll, Lo 1970a) t-ike-t $\sim$ t-ikae-t '¿qué?'.

(12) L (M) mi-ma 'aquél', me-oto 'aquellos'; V (Lo 1970b) -m (-V), - me -mi (-C) ‘3a. p. objeto'.

(13) L (M) $\boldsymbol{t}\left(\boldsymbol{t}^{\prime}\right) \boldsymbol{e}$ - 'deíctico de distancia mínima', $\boldsymbol{t}\left({ }^{\prime}\right) \boldsymbol{i}-\boldsymbol{t}\left({ }^{\prime}\right) \boldsymbol{a}, \boldsymbol{t}\left({ }^{\prime}\right) \boldsymbol{i}-\boldsymbol{t}\left({ }^{\prime}\right) \boldsymbol{e}$ 'éste'; V (Lo 1970a, 1970b) $\boldsymbol{t}$ 'e, $\boldsymbol{t}$ '- 'deíctico de distancia media', (L1, HB 1967, Lo 1970a, 1970b) $\boldsymbol{t}$ 'e-t(e) 'este, ese'.

\subsection{Comparativos/aditivos}

(14) L (M) m-eke-p 'así también', 'también asî'; V (AV, Pe) umkel 'también'.

(15) L (M, AV) m-eke-to 'así como', 'como'; V (Lo 1970a) -mket- -mkit- 'aspecto habitual'.

Transformado en sufijo verbal en V.

(16) L (M, AV) t(')-eke-si, $\boldsymbol{m}$-eke-si 'asî'; V (Pe, Ll, HB 1998) eset set 'como, tal, así'.

Con respecto a L $\boldsymbol{m}$ - en (14), (15) y (16), cf. (12); para t(')-, cf. (13). L -eke- es el mismo elemento que aparece en (14) y (15). 


\subsection{Sufijos casuales (locativos)}

(17) L (M, AV) -le 'hacia, en, desde', 'si, cuando'; V (AV, Lo 1970a, 1977) -lat -let 'en, por, hacia, a'.

(18) L (M) -ta 'por, desde'; V - $\boldsymbol{t}$ final del sufijo -lat -let 'en, por, hacia, a', cf. (17)..

En la bibliografía se menciona un presunto sufijo locativo $\mathrm{V}$-at. Esto se debe a que AV (513) segmentaron en un Padrenuestro de esta lengua la forma lauè - $\mathbf{l}$-àt "alturas" + artículo + "en". LQ $(1895: 82,107)$ comparó L -ta y este supuesto sufijo V -at. "en", lo que luego fue repetido por Greenberg (1987: 303, ítem gramatical n 49). Pero el "articulo" V $\boldsymbol{l}$ - no existe, siendo el sufijo -lat sincrónicamente indivisible.

(19) L (M) -ya 'locativo'; V (HB 1998) $\boldsymbol{h}$-ya 'ahí, allá' (he- es un demostrativo documentado por Ll, HB 1967, Lo 1964).

\subsection{Otros funcionales}

(20) L (M) t(')-ayule, m-ayule 'por, a causa de'; V (Lo 1970a) yubel hubel 'en seguida'. L-ayule <*-ayuwule; para t'(')- y m-, cf. (13) y (12). Para la correspondencia semántica, cf. Castellano luego con el sentido de 'por lo tanto'.

(21) L (M) yawo 'junto'; V (HB 1998, Lo 1970a, 1977) hube ube 'juntos', 'con'.

\subsection{Sufijos de plural}

(22) L (M) - $\boldsymbol{l}, \boldsymbol{-} \boldsymbol{e l}, \boldsymbol{-} \boldsymbol{l} \boldsymbol{e}, \boldsymbol{- i l}, \boldsymbol{- \boldsymbol { y } \boldsymbol { l }}$ 'plural' (en el pronombre 'vosotros', cf. (5), y en sustantivos que se refieren a personas), $\boldsymbol{t}-\boldsymbol{t}$-il 'plural' (en dos sustantivos referidos a personas, cf. (24)); V -le, -lem, -lom 'plural' (en sustantivos).

(23) L - $\boldsymbol{n}$ 'plural' (en marcas de persona sujeto/posesivos: $-\boldsymbol{c} \boldsymbol{e}-\boldsymbol{n}$ '1a. p. pl.', cf. (1), - $\boldsymbol{w a} \boldsymbol{a} \boldsymbol{n}$ '2a. p. pl.', cf. we 'tú', -pa-n '3a. p. pl.', cf. (6)); V -m final del sufijo-lem, -lom 'plural' (de sustantivos), cf. (22).

(24) L -oto 'plural de deícticos', -t-il 'plural' (en sustantivos que se refieren a personas, cf. (22)); V - $t$ final del sufijo de 1a. p. plural inclusivo, cf. (9), y en el correspondiente pronombre nita-t 'nosotros (inclusivo)', de la raíz que aparece en nike-pa $\sim$ nite-pa 'señor', cf. (112).

(25) L -s final del pronombre de 1a. p. sing.; V -s 'plural (de 1a. p.)', cf. (2).

La comparación de (1) y (2) muestra que $\mathrm{V}-\boldsymbol{s}$ es originariamente un sufijo de plural, que solamente ocurre en el pronombre de 1a. persona, como notó Lo (1964: 156), aunque esta autora atribuyó tal sufijo a una "probable influencia española", lo que resulta insostenible a la luz de la coincidencia formal en L (si bien en esta lengua habría ocurrido un cambio semántico de `nosotros' a ‘yo').

\subsection{Afijos nominalizadores}

(26) L (HB 1967) -p; V (HB 1967, Lo 1970a) -p - pa - pe "nominalizador denominal (nombres de objetos poseíbles, incluyendo nombres de parentesco)', 'adjetivizador'. 
(27) L (M) -pe (con reduplicación de la raíz) 'sufijo nominalizador (locativo-instrumental)';

V (Lo 1970a, 1970b) pe - $\sim \boldsymbol{p}$--, -pe 'afijos nominalizadores (ubicativo)'.

(28) L (HB 1967) - $\boldsymbol{t}$; V (HB 1967, Lo 1970a) $-\boldsymbol{t}$, -te 'sufijo nominalizador denominal'.

\subsection{Marcadores de tiempo/modo}

(29) L (M) ate 'pasado remoto'; V (Lo 1970a) ete $\sim$ et 'ya'.

(30) L (M) -le 'subjuntivo', 'gerundio'; V (Lo 1970a, 1970b) - $l$, - $\boldsymbol{e l}$ 'gerundio'.

(31) L: (M) - $\boldsymbol{n}$ 'futuro'; V (Lo 1970b) -noh 'futuro remoto'.

(32) L (M) -n(i) 'pasado reciente'; V (Lo 1970a, 1970b) -n 'aspecto perfectivo'.

(33) L (M) - pe; V (Lo 1977) p- (-V), pe- (-C) 'exhortativo'.

\subsection{Prefijos causativos/transitivizadores y clasificadores verbales}

(34) L me-; V (AV, Lo 1970b) um- 'causativo'.

L documentado en un solo ejemplo: $\boldsymbol{m e}$-ne- 'traer', cf. (172), de -ne- 'venir', cf. (173).

(35) L $\boldsymbol{n a}-, \boldsymbol{n e}-; \mathrm{V} \boldsymbol{n}$ - 'causativo'

Ejemplos L: (M) na-as- 'hacer enfadar', de as- 'enojarse'; $\boldsymbol{n a}$-maka-s- 'engordar', de maka 'grasa', maka-p 'gordo'; na-na ?t- 'contradecir', de na ?t- 'responder'; ne-kekes'gobernar', de kekes- 'caber, entrar bien'; ne-key-c- 'enfriar soplando', de $\boldsymbol{k} \boldsymbol{e y -}$ - 'hacer o tener frío', etc. El prefijo V está documentado en un solo ejemplo: (HB ms) n-a łe 'cocinar', de at-ałe, a te 'hervir', cf. (160).

(36) L (Lársen en M: 10, LQ 1894b) nik-, $\boldsymbol{n i}$-; V inu-, un-, $\boldsymbol{n i -}$ 'con las manos'. Para el V, cf. (174), (175), (176).

(37) L s-; V ah- 'causativo'/'transitivizador'.

Ejemplos L: (M) s-uyom- 'embutir', de uyom- 'entrar muchos'; $\boldsymbol{s}$-mut- 'zambullir a otro', de mut- 'zambullirse', s-awey- 'perdonar', de awey- 'apiadarse', etc. Para el V, cf. las series (164), (167), (181), (182), (183), (184), (196).

(38) L (LQ 1894b) yak(')s-, yek(')s- 'con los pies'; V (Ll, Lo 1970b) yok'o- 'pisar'.

\subsection{Otros afijos verbales}

(39) L le-; V la- 'prefijo de verbos de posición o movimiento' (?).

Ejemplo L: (M) co-le-ki- 'levantarse' (con incorporación de co 'arriba'), de $\boldsymbol{k i}$ - 'parar'. Ejemplo V: (Lo 1970a) la-pim- 'pararse', de pim- 'parado'.

(40) L (M, LQ 189b) -ton, -to "participio de presente", 'agente'; V (Lo 1970a, 1970b, 1977) -it, $-\boldsymbol{t}$ 'relativo', 'participio'. 


\subsection{Sufijo privativo y negación}

(41) L (LQ 1894b) - $\boldsymbol{k}$ (') $\boldsymbol{o}-\boldsymbol{p}$; V (Lo 1970a) -rop 'sin' .

Posiblemente L - $\boldsymbol{k}($ ') - y V - $\boldsymbol{r}$ - sean generalizaciones de consonantes originariamente antihiáticas.

(42) L (M, AV) uye ‘no, ninguno', 'sin'; V (Pe, Ll, Lo 1970a, 1970b) iya iye 'no hay'.

\section{COGNADOS LÉXICOS}

Presento los cognados léxicos Lule-Vilela divididos en once campos semánticos: naturaleza inanimada; vegetales, sus partes y productos; animales y sus productos; partes del cuerpo humano y animal; relaciones familiares y sociales; cultura material; cultura espiritual y vida psíquica; cualidades; número y cantidad; relaciones espaciales y temporales; acciones y estados.

\subsection{Naturaleza inanimada}

(43) L (M) a-cupu; V (Pe, Ll, HB 1967, 1998) sukeh, suk'ek, suq'el, suq'os 'ceniza', (Ll, MC 1968) suqu 'trozo de carbón'.

(44) L (M) a-kso; V (HB 1967) tusu 'humo'.

En L con disimulación $* t s>k s$.

(45) L (M) a-tiła 'barro, pantano'; V (JG, Pe) ite t 'río'.

L $\boldsymbol{a}$ - en (43), (44) y (45) parece ser una incorporación de (M, Fe) $\boldsymbol{P a} \sim \boldsymbol{a}$ 'tierra'.

(46) L (M) away- 'amanecer'; V (JG, Ll) p-awe 'día, luz, claridad'.

La forma $\mathrm{V}$ incorpora el prefijo ubicativo $\boldsymbol{p}(\boldsymbol{e})-$, cf. (27).

(47) L (M) awi 'campo, pampa, vega'; V (Pe, Ll, HB 1998, MC 1965, Lo 1970a, 1970b, 1977) aye 'campo'.

(48) L (M, Fe, AV) ini 'día', 'sol'; V ini- in- *‘día'.

$\mathrm{V}$ solamente en un compuesto, cf. (133).

(49) L (M) $\boldsymbol{a} \boldsymbol{k}$ (')op, $\boldsymbol{a k}$ (')ep 'luz (de astro)'; V (JG, Fo, Pe, Ll, LN) $\boldsymbol{k}$ 'opi 'luna' .

(50) L-me * 'jugo'; V (JG, Fo, Pe, Am, Ll, HB 1998, Lo 1970a) ma 'agua'.

L sólo en compuestos como (M) ine-me 'leche', cf. (95), yana-me (L yana 'esp. de abeja'), yala-me (cf. (91)), ane-me, awokoc-me, kilili-me 'distintas clases de miel'. (51) L (M) o Po 'polvo'; V (Ll) hu 'arena'.

(52) L (M) u tum- 'llama, llamear'; V (Lo 1970a) ah-lim-lim- 'llamarada'.

(53) L (M) usi isi 'pozo'; V (Lo 1970a, 1977) hi- 'cueva'.

(54) L (M) uy ?o; V (JG, Ll, HB 1967) uy, uya-t 'noche'.

\subsection{Vegetales, sus partes y productos}

(55) L (M) an-ca-p an-c-p 'hoja'; V (JG, Ll, HB 1967, MC 1965) ane 'hierba'. La forma L parece incorporar ca?- 'verde', cf. (128). 
(56) L (M) cele- 'carbón', V (J, Pe, Ll, HB 1967, 1998, MC 1965, Lo 1970a, 1977)

kire tire 'árbol, madera, leña, bosque, palo, monte, carbón'.

(57) L (M) eles 'espina'; V (HB 1998, Lo 1970b, MC 1968) ilis 'tunita'.

(58) L (M) e-sic 'rama'; V (MC 1965) seye 'tallo, rama'.

L al parecer con incorporación de $\boldsymbol{e}$ 'palo, madera, árbol'.

(59) L (M) kesi; V (MC 1965) kiti-kiti ‘cadillo'.

(60) L (M) kowil-po-po 'azucena'; V (MC 1965) kobil 'cebolla (y otras plantas)'.

(61) L (M, Fe) na ła 'hierba'; V (Ll, MC 1965) niłe 'hoja'

(62) L (M) ne-p 'espiga'; V (MC 1965) na-pe 'vaina o legumbre'.

(63) L (M) noko 'chaguar'; V (HB 1998, Lo 1970a, MC 1968) ma-noqo 'chaguar, cardo'.

(64) L (M) pis; V (MC 1965) ruk-pihe 'raíz'.

(65) L (M) wala 'quebracho colorado'; V (Ll, MC 1965) walek 'quebracho blanco'.

(66) L (M) waya 'algarrobo verde negro y su fruta'; V (MC 1965) baye 'mistol'.

(67) L (M) yo ?; V (Ll, HB 1967, MC 1965) yo 'calabaza, zapallo'.

\subsection{Animales y sus productos}

(68) L (M) akoko; V (MC 1995) oqoqo 'sapo'. Posible onomatopeya.

(69) L (M) amil akwa; V (L1, HB 1998, MC 1995) amet 'avispa lechiguana'.

(70) L (M) ayan; $\mathrm{V}$ (Ll, MC 1965) ayem 'iguana'.

(71) L (M) cala kiki-p-; V (L1, MC 1995) sale kitkit 'lechuza'.

(72) L (M) $k a$ Pya Pe 'serpiente'; V (Fo, Ll, MC 1995) aqe 'víbora, serpiente'.

(73) L (M, Fe) koks 'ciervo'; V (JG, Am, Ll, HB 1967, 1998, MC 1995) kihi tihi ('ciervo' >) 'caballo'.

(74) L (M) kopas 'avispa'; V (JG, Ll, MC 1995) qahpa 'avispas y abejas en general'.

(75) L (M) kopo 'quirquincho'; V (MC 1995) qapi 'mataco o tatú bolita'.

(76) L (M, Fe, J) kule ‘perro'; V (JG) oqol ‘zorro', (J, Fo, Pe, Ll, LN, MC 1995) wan-oqol 'perro' (compuesto con wan- 'casa', cf. (116)).

(77) L (M) kum; V (Ll, MC 1995) kum 'caracol'.

(78) L (M) ma-ma 'carcoma, polilla'; V (MC 1995) mah `larva, gusano'.

(79) L (M) masac; V (Pe, Am, Ll, Lo 1970a, MC 1995) masak masaq 'nutria (roedor acuático)'.

(80) L (M) pin-pin; $\mathrm{V}$ (Ll, MC 1995) pim-pim 'pulga'.

(81) L (M) poko- 'apolillarse'; V (L1, MC 1995) poqo-poqo-li 'mariposas en general'.

(82) L (M) su 'especie de hormiga'; V (JG, Ll, MC 1995) su-su-li 'hormiga'.

(83) L (M) s Pala; $\mathrm{V}$ (Fo, Ll, MC 1995) sale 'ciervo'.

(84) L (M) t(')a; V (Ll, HB 1967) are 'huevo'.

(85) L (M) $\boldsymbol{u} ; \mathrm{V}$ (Ll, MC 1995) ba-lit 'langosta'. L $\boldsymbol{u}<* \boldsymbol{w} \boldsymbol{u}$.

(86) L (M) ulus; V (Ll, HB 1998, MC 1965, 1995) wulus, gulus 'rata, ratón'.

(87) L (M) u tuku 'garrapata grande'; V (Lo 1970a) walewagwak 'garrapata'.

(88) L (M) upcan 'lagartija'; V (Ll) ipesoksok 'lagarto'.

(89) L (M) walamal; $\mathrm{V}$ (MC 1995) waleme 'vizcacha'.

(90) L (M) wokat(')a 'pava de monte, gallina, gallo'; V (J, Ll, MC 1995) wokare okare 'gallina'. 
(91) L (M) yalam akwa 'abeja moro moro'; V (MC 1995) yaleme mop ‘larva del gusano del pindó'.

\subsection{Partes del cuerpo humano y animal}

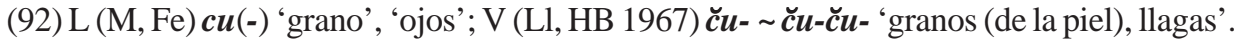

(93) L (M) $\boldsymbol{e}$ tu 'pie'; V (Pe) $\boldsymbol{u}$ tu- 'vaso de caballo'.

(94) L (M) ewe; V (Ll, HB 1967) awo 'sangre'.

(95) L (M, Fe) ine 'teta(s)'; V (HB 1998, HBR) ine-, ina- 'amamantar'.

(96) L (M, Fe) is 'mano'; V (JG, Fo, Pe, Ll, HB 1967, HBR, Lo 1977) isi- 'mano, dedos'.

(97) L (M, Fe) $\boldsymbol{k}$ (') $\boldsymbol{a}$ 'boca'; V (HB 1967, 1998) $\boldsymbol{k}$ 'o 'agujero'.

(98) L (M) kanap; V (Ll) kanake 'hígado'. L -p podría ser un sufijo.

(99) L (M) ko ?- 'útero'; V (Ll) kowe 'vagina'.

(100) L (M) ley- 'anca'; V (Ll) lahe 'nalgas'.

(101) L (M, Fe) łu 'diente'; V (JG, Pe, MC 1965) tu- 'diente', 'grano de maíz' .

(102) L (M) nawo- 'cresta'; V (Fo, Pe, Ll, HB 1998, MC 1965) nahu-, noho- 'cabello, pelo, hoja'.

(103) L (M, Fe) nus 'nariz, hocico'; V (Fo, Pe, Ll, HB 1967) nihi- 'nariz'. Posible onomatopeya. (104) L (M) pis- 'vena, nervio'; V (Ll) pis- 'arteria, vena'.

(105) L (M) su ?kwe 'pelo de animal, lana'; V (HB ms) sukwiye- 'esquilar'.

(106) L (M, Fe) $\boldsymbol{u}$-; V (Pe) $\boldsymbol{i}$ - 'cuello'.

(107) L (M) u 'voz'; V (L1, Lo 1970a, 1970b) oho- 'decir'.

\subsection{Relaciones familiares y sociales}

(108) L (LQ 1894b: 319) eme 'esposa'; V (JG, Ll, HB 1998, Lo 1970a, 1970b, 1977) gima 'cónyuge', gima- gime- gim- geme- 'casarse'.

La forma L sólo está documentada formando parte de sintagmas: $k$ we-p eme-p 'nuera (de suegros vivos)', kwe eme apa-p 'nuera (de suegros fallecidos)', etc.

(109) L (M) kiła kila 'mujer joven', kili- 'hembra'; V (JG, Pe, Am, Ll, LN, HBR, Lo 1970a, 1977) kiła kiłe 'mujer, hembra'.

(110) L (M, Fe, D) kwe 'hijo, niño'; V (D, JG. Fo, Ll, Lo 1970a, 1977) ina-qe 'hijo'. La forma $\mathrm{V}$ es un compuesto con ina- 'amamantar', cf. (95).

(111) L (M) kwe-lu(t), lu-kwe 'hijo varón'; V (Ll, HB 1967, MC 1995) holet 'macho, varón' . (112) L (M) nikoyo; V (Fo, Ll, HB 1967, Lo 1970a) nite-pa nike-pa 'señor'

(113) L (M, Fe, D, AV) $\boldsymbol{p} \boldsymbol{e} ; \mathrm{V}$ (JG, Pe, HBR) o- 'padre'.

(114) L (M, Fe) pele 'hombre', 'persona (indígena)'; V (Lo 1970a) pile pila 'hombre joven'.

(115) L (M, Fe) wakal; V (HB 1967) wakal 'mujer'. 


\subsection{Cultura material}

(116) L (M, Fe) enu 'casa', 'ciudad, pueblo'; V (JG, Pe, Am, Ll, HB 1967, 1998, Lo 1970a, 1970b) wane 'casa, toldo'.

(117) L (M) $\boldsymbol{e} \boldsymbol{t} \boldsymbol{u} ; \mathrm{V}$ (Pe, Ll, HB 1967, Lo 1970b, 1977) a tue a tu 'cuchillo'.

(118) L (M, Fe) su-ma 'chicha'; V (JG, Fo, Pe, Ll, HB 1998, Lo 1970a) suk'e 'chicha, aloja', sup- 'emborracharse, estar borracho'.

(119) L (M) uya 'bolsa'; V (Pe, Ll) iyah 'hamaca'.

(120) L (M) yu-pa 'calabazo, vaso, mate, taza'; V (JG, Am, Ll, HB 1967, 1998) yo-pe $\sim \boldsymbol{y o}-\boldsymbol{p}$ 'cuenco, olla' (derivado de (67)).

\subsection{Cultura espiritual y vida psíquica}

(121) L (M) e Pnup, $\boldsymbol{e}$ ?nup-; V (L1, MC 1968, Lo 1970a) wanaq- 'juego, jugar'.

(122) L (M, Fe) ice, ice-t 'alma, espíritu', 'corazón'; V (JG, AV, Pe, LN, HB 1998, Lo 1970a)

Gose Gos 'alma, espíritu, demonio', 'pecho', 'corazón'.

(123) L (M) un- 'sabor'; V (Ll, Lo 1970b) hini- hin- 'dulce'.

(124) L (M) usi 'miedo'; V (Ll, HB 1998) Gose- 'asustar, asustar'.

(125) L (M, D, AV) wet(i-) 'nombre, nombrar'; $\mathrm{V}(\mathrm{D}, \mathrm{AV}, \mathrm{HB}$ 1967) wate 'nombre'.

\subsection{Cualidades}

(126) L (M) alu-; V (Ll, HB 1967, Lo 1970a) alu- aru- 'calor, caliente'.

(127) L (M) cakweci- 'azul, verde'; V (Ll, HB 1967, 1998, MC 1965) čakotti- čaktti- 'azul, violeta, verde'. L -ci- $(=-t s i-)<*-t t i-$.

(128) L (M) $\boldsymbol{c a}$ ?- sa ?- 'verde', 'amargo'; V (JG, Ll, HB 1967, 1998) sa-ne- sa-na- sa-nana- 'verde', 'amarillo'.

La forma V incorpora ane 'hierba', cf. (55).

(129) L (M, Fe) cele-; V (JG, Pe, Ll, HB 1967) kirimi- 'negro'.

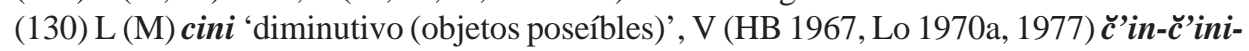
'pequeño'.

(131) L (M) eci- '(estar) bien, bueno'; V (Ll, HB 1967, 1998, Lo 1970a) ati- 'bien, bueno, saludable, hermoso'.

(132) L (M) ik'eto 'horrible'; V (Ll, Lo 1970a) ik' 'et 'feo, malo, enfermo'.

(133) L (M) in-le 'ayer'; V (HB 1967) in-ta ini-ła 'viejo'.

Según HB (1967: 29-30) el significado original de la forma V sería “crecido, largo en días".

(134) L (M) ka-kala- 'mancha (natural en la piel de un animal); V (MC 1995) qolo-p '(color de piel) bataraz, manchado'.

(135) L (M) kolos- 'torcido'; V (MC 1965) kulus 'enroscado'. 
Viegas Barros: Evidencias de la Relación Genética Lule-Vilela

(136) L (M) mo-p 'harina'; V (Pe, HB 1967) moh- mo- 'fino'.

(137) L (M) mo P-le 'hace mucho tiempo', 'antiguamente', 'ya (hace tiempo)'; V (MC 1965) mo-p 'abuelo'.

(138) L (M, AV) oseyu; V (Ll, HB 1967) gusi- gus- 'malo'.

(139) L (M, Fe) $\boldsymbol{p}$ (')o P-; V (JG, Pe. Ll, HB 1967, 1998, Lo 1970b) p’o-, o- 'blanco'.

(140) L (M) pulum 'grande, grueso'; V (HB 1967) olem- 'grande'.

(141) L (M) saps 'delgado'; V (J, Ll, MC 1995) sepi ‘serpiente'.

(142) L (M) sces-sces ce-ces 'diminutivo'; V (Pe, Am, Ll, HB 1998) ahtis ahkis 'pequeño'.

(143) L (M) stonot- ‘(ser) enano'; V (Ll, HB 1998, Lo 1970a) tone ‘corto'.

(144) L (M, J) yos ‘ampalagua, una especie de víbora'; V (Ll, Lo 1970a, 1977) yosi- yos'ser flaco'.

\subsection{Número y cantidad}

(145) L (M) alap 'unión', alapea 'uno, único'; V (Lo 1970a) lapge ‘todo' .

(146) L (M) ewi-; V (Pe, Am. Ll. HB 1967, MC 1965) owe $\sim \boldsymbol{o} \boldsymbol{e}$ 'mucho', -owe 'colectivo (con nombres de plantas)'.

(147) L (M) lokwep; V (Pe, Am, Ll, Lo 1970a, MC 1968) pukewale ‘cuatro'.

Metátesis en una de las dos lenguas..

(148) L (M) oca 'lleno de..., que tiene mucho...'; V (L1, HB 1967, Lo 1970a) otie eti 'mucho, muy'

(149) L (M) tam-lip; Vi (Pe, Am, Ll, Lo 1977, MC 1968) nipetwey 'tres'.

L tamlip disimilado de *tamnip. El final V -twey queda sin explicación.

(150) L (M) tam-op; V (Pe, Am, Ll, Lo 1970a, 1977, MC 1968) uke 'dos'. Cf. también L (M) wop- "aparejar”, es decir, 'poner de a dos'.

(151) L (M) -t(')u 'colectivo (con nombres de plantas)'; V (HB 1967, Lo 1970a) ru-p ru-pe $\sim$ lu-pe 'mucho, muy'.

\subsection{Relaciones espaciales y temporales}

(152) L (M) an Pa(-) '(estar) hacia arriba'; V (Ll, HB 1967, Lo 1977) an- 'subir'.

(153) L (M) an Pop-le; V (HB 1998, Lo 1970a) nipe-le ‘delante, primero'.

(154) L (M) ele-; V (HB 1998) eli- 'acostarse'.

(155) L (M) ew-tita, V (HB 1998, Lo 1970a) wa-te, wa-he 'ahora'.

Tanto L-tita como V -te, -he son deícticos de distancia mínima.

(156) L (M) la- 'subir'; V (JG, Ll, HB ms., Lo 1977) laoe lawe 'arriba'.

(157) L (M) lep- 'entrar'; V (HB 1967) lopa ‘dentro de'.

(158) L (M) wa ? 'detrás'; V (L1, HB 1998) u- 'cola'. 


\subsection{Acciones y estados}

(159) L (M) ac (')isa-; V (Ll) at(')iso- 'estornudar'. Posible onomatopeya.

(160) L (M) ale- 'arder'; V (Ll, Lo 1970a) a te-, at-a te- 'hervir'. Cf. (126).

(161) L (M) cic- 'amarrar, anudar'; V (Ll, HB 1998) siki-, sik'i-, sikik- 'atar'.

(162) L (M) cililis- 'caer(se), estar por caer'; V (Lo 1970a, 1977) kit-ki- 'caer(se), acostarse'.

(163) L (M, AV) cu-k-; V (AV, Ll) $\boldsymbol{i}$-c $\breve{c} \boldsymbol{u}$ - 'besar'.

(164) L (M) $\boldsymbol{e}$ - 'ver, mirar, hallar'; V (Ll, Lo 1970b) ah-aye- a-aye- 'encontrar'.

(165) L (M) eyes, eyes- '(decir un) secreto'; V (Lo 1970b) yah-yah- 'callarse'.

(166) L (M) ice- ; V (Lo 1970a) keh- 'crecer'.

(167) L (M) i ?o-; V (Ll) ah-eyu 'tardar'.

(168) L (M) $\boldsymbol{k a} \boldsymbol{a}$; V (HB 1998, Lo 1970b, 1977) $\boldsymbol{k} \boldsymbol{a}$ - $\boldsymbol{k} \boldsymbol{e}-\sim \boldsymbol{k i}$ - 'ir, andar'.

(169) L (M) $k$ ay-; V (Ll, HB 1967, Lo 1970a) laqay laqe 'comer'.

(170) L (M) kalip- 'brillar'; V (Ll) kelep-lepa 'brillo' (reduplicación incompleta).

(171) L (M) $\boldsymbol{k}$ (')e-k(')e-s- ; V (Lo 1970b) k'at-k'at- k'ate-k'at 'caber bien, ajustar'.

(172) L (M) me-ne-; V (Lo 1970a, 1970b) um-ne- um-na- 'traer'.

(173) L (M, AV) $\boldsymbol{n} \boldsymbol{e}-, \mathrm{V}$ (AV, Pe, HBR, Lo 1970b, 1977) $\boldsymbol{n} \boldsymbol{e}-\sim \boldsymbol{n} \boldsymbol{o}-\sim \boldsymbol{n} \boldsymbol{a}-\sim \boldsymbol{n i}$ - 'venir'.

(174) L (M) ni-cikips- 'arañar con las manos'; V (Ll, MC 1965) inu-sisi-, un-sisi- 'arañar, rascar'.

(175) L (M) ni-culu-kukuc- 'tener o hacer cosquillas', cul- 'comezón, cosquillas'; V (Ll) nisula- 'cosquillas'.

(176) L (M) nik-ol- 'enredar'; V (MC 1965) nikolom 'enredaderas'.

(177) L (M) o Po-; V (Ll) ohom- 'tos, toser'. Posible onomatopeya.

(178) L (M) pok-; V (Lo 1977) um-hoq- 'cavar'.

(179) L (M) p (')ululus- 'dar vuelta'; V (Ll, Lo 1970a) p'iligi- 'darse vuelta'.

(180) L (M) se-; V (Ll, Lo 1970a) ehe- 'llorar'.

(181) L (M) sip- 'considerar', 'pensar'; V (Ll, Lo 1970a) ah-sap- 'conocer, saber'.

(182) L (M) s-nus- 'convidar'; V (Lo 1977) ah-nih- 'invitar'.

(183) L (M) s-telelet- 'rodar'; V (Ll, MC 1968) ah-tolelite 'dar vuelta(s)'.

(184) L (M) ta-cil-; V (MC 1968) ah-čil-čil- 'salpicar'.

(185) L (M) tun-, tum-; V (HB 1967) tum- 'terminar, acabar, detenerse'.

(186) L (M) $\boldsymbol{t}($ ') $\boldsymbol{a}$ - 'recoger plantas, segar'; V (L1, Lo 1964, 1970a, 1977) are- 'tomar, agarrar'.

(187) L (M) t')i-; V (Lo 1965, 1970b) ri- ra- ro- 'hacer'.

(188) L (M) us- Pus-; V (Ll) gisle- 'oler'.

(189) L (M) was-; V (L1, HB 1967, Lo 1970a, 1970b) bise- bis- 'pedir'.

(190) L (M) wene- $\boldsymbol{k}$ - 'comprar'; V (Ll) wene-l- 'vender'.

(191) L (M) wes- 'comer vegetales'; V (JG, Pe, Ll, HB 1998, Lo 1970a, 1970b, 1977) wahewah- 'comer', wahe $\sim$ wah 'comida'.

(192) L (M) wi-; V (Pe, Ll, Lo 1954) wisak- 'dormir'.

(193) L (M) wos- 'llegar (sujeto singular)'; V (Lo 1970b) hos- 'llegar'.

(194) L (M) yap-toy-; V (HB ms.) yeptu- 'sacar, quitar'.

(195) L (M) yasi- 'quedarse'; V (AV, Pe, Ll, Lo 1970a, 1970b) yasi- 'sentarse', 'estar (sentado)'.

(196) L (M) $\boldsymbol{y} \boldsymbol{a}$ ?u- 'ir, andar, caminar, pasearse'; V (Ll, HB 1998, Lo 1970a) ah-yuhe- 'andar'.

(197) L (M) yep- 'dar'; V (Lo 1970b) yip- 'repartir, dar, servir'.

(198) L (M) yepit-; V (Ll) eyepi- 'estirar(se)'. 
(199) L (M) yеp-sumu- 'remojar'; V (Lo 1977) yeču- 'mojar, remojar'.

(200) L (M) yuys- (o iwis-, o ywis-) 'atender, oir', V (Pe) isi- 'oídos'.

\section{CONCLUSIONES}

En el presente trabajo se han presentado todas las evidencias en favor del parentesco Lule-Vilela que hasta el momento he podido encontrar.

Se ha mostrado que existen correspondencias fonológicas regulares, incluyendo algunas que no son triviales (como las que se esperarían en casos de préstamos relativamente recientes), p. ej.:
$\mathrm{L} \emptyset: \mathrm{V} \mathbf{g}$.
$\mathrm{L} \mathbf{p}: \mathrm{V} \emptyset, \mathbf{h}$.
$\mathrm{L} \mathbf{s}: \mathrm{Vh}$
L p : Vk.
L c : V k, k $\sim \mathbf{t}, \mathbf{t}$.
Lu : Vi,e.

También se demostró que hay por lo menos tres docenas y media de similaridades gramaticales, a las que se pueden agregar otras semejanzas estructurales, tales como:

- la existencia en ambas lenguas de raíces distintas para el singular y el plural en algunos verbos, sobre todo de movimiento o de posición: L hos- (sing.) frente a wal- (pl.) 'llegar', oc- (sing.) frente a yewti- (pl.) 'salir', lep- (sing.) frente a uyom- (pl.) 'entrar', etc.; V sa- (sing.) frente a le $\mathbf{~} u \boldsymbol{h}$ - (pl.) 'entrar', yasi- (sing.) frente a lil- (pl.) 'estar', etc.,

- la derivación por medio de reduplicación (completa o no) con un significado atenuativo: L ol-olom- 'hablar poco' de olom- 'hablar', si-si- 'haber poco' de si- 'haber', lu-lu- 'tibio' de alu- 'caliente', etc.; V wasa-sa-let "tejidito" de wasa- 'tejido', $k$ as-kas-li "amarguito" de kas- 'amargo', etc. (V -let -li es un sufijo diminutivo);

- los prefijos verbales instrumentales, por lo general formalmente muy distintos de

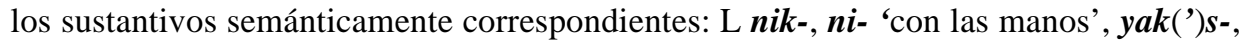
$\boldsymbol{y e k}(') s-$ 'con los pies', tak-, ta- 'con herramienta o palo', etc., cf. is 'mano'; ełu 'pie'; $\boldsymbol{e}$ 'palo'; en $\mathrm{V}$ ningún autor los menciona, pero un estudio atento de la documentación de esta lengua muestra la existencia de los prefijos inu-, un-, ni- 'con las manos', it- 'con la boca', etc., cf. V is(i)- 'mano', ge- 'boca'.

En cuanto al léxico, las series cognadas incluyen un número considerable de términos del llamado vocabulario "básico" o "nuclear", p. ej.: 'noche' (54), ‘sangre' (94), ‘mano' (96), 'diente' (101), 'padre' (113), 'mujer' (115), 'ir' (168), 'venir' (173), ‘hacer' (187), etc.

Finalmente, la admisión del parentesco lule-vilela provee una aclaración etimológica satisfactoria para ciertas formas que se encuentran en una de las dos lenguas solamente como miembros de compuestos o en sintagmas aparentemente cristalizados, p. ej. L eme 'esposa', cf. (108), o el comienzo de V in(i)-ła 'viejo', cf. (48) y (133).

Por todo ello, considero que la hipótesis de parentesco lule-vilela puede darse por demostrada, si bien restan todavía por realizar un par de tareas cruciales: primero, expandir tanto como sea posible la base comparativa, y segundo, intentar la reconstrucción de la fonología del Proto-Lule-Vilela. 


\section{REFERENCIASBIBLIOGRÁFICAS}

ADELAAR, W. F. H. 1991. The endangered languages problem: South America, Endangered Languages, ed. by R. H. Robins y E. M. Uhlenbeck. Published with autorithy of the Permanent International Committee of Linguists (CIPL), Oxford/Nueva York: Berg: 45-91.

ADELUNG, J. Ch y J. S. Vater. 1813. Mithridates oder allgemeine Sprachenkunde mit dem Vater Unser als Sprachprobe in beynahe fünfhundert Sprachen und Mundarten. Dritter Theil. Sweyte Abtheilung. Berlin: Vossischen Buchhandlung: 310-708.

AMBROSETTI, J. B. 1894. Apuntes sobre los indios chunupíes (Chaco austral) y pequeño vocabulario, Anales de la Sociedad Científica Argentina, Buenos Aires, 37: 151-160.

BALMORI, D. 1998. Clemento Hernando Balmori. Los textos de un lingüista. Sada-A Coruña: Ediçoes do Castro.

BRAVO, D. A. 1975. Diccionario quichua santiagueño-castellano. Prólogo de A. Colombres, Buenos Aires: EUDEBA.

DOBRIZHOFFER, M. 1968. Historia de los Abipones, Resistencia: Univ. Nac. del Nordeste, Fac. de Humanidades, trad. de C. Cedoya de Guillén, vol. II.

FABRE, A. 1998. Manual de las lenguas indígenas sudamericanas, München-Newcastle: LINCOM EUROPA, 2 vol.

FONTANA, L. J. 1881. El Gran Chaco, Buenos Aires: Imp. de Ostwald y Martínez.

GILIJ, F. S. 1965. Ensayo de Historia Americana o sea Historia Natural, Civil y Sacra de los Reinos y de las Provincias Españolas de Tierra Firme en la América Meridional, Tomo III. Trad. de A. Tovar, Caracas: Fuentes para la Historia Colonial de Venezuela, Biblioteca de la Acad. Nac. de la Historia, 73. Ed. original: Roma, 1782.

GREENBERG, J. H. 1987. Language in the Americas, Stanford: Stanford Univ. Press.

HERNANDO BALMORI, C. (1957). Notas de un viaje a los Tobas, Revista de la Universidad, La Plata, 2: 23-36.

.(1959). Doña Dominga Galarza y las postrimerías de un pueblo y una lengua, Revista de la Universidad, La Plata, 9: 85-98.

.(1967). Ensayo comparativo lule-vilela: sufijos -p y -t. Con un breve texto vilela, Estudios de área linguiística indígena. Buenos Aires: Univ. de Buenos Aires, Fac. de Filosofía y Letras, Centro de Estudios Lingüísticos: 9-32..

.(1998). Diccionario Vilela a, b, c. En Balmori (1998: 139-252).

.(1963). ms. Chaco 1963.

HERVÁS, L. 1800. Catálogo de las lenguas de las naciones conocidas y numeración, división y clases de éstas, según la diversidad de sus idiomas y dialectos. Vol. 1, Lenguas y naciones americanas. Madrid: Imp. de la Adm. del Real Arbitrio de Beneficencia.

JOLÍS, J. 1972. Ensayo sobre la historia natural del Gran Chaco. Traducción de M. L. Acuña. Estudio preliminar de J. A. Maeder. Resistencia (Chaco): Univ. Nac. del Nordeste, Fac. de Humanidades, Inst. de Historia. Ed. original: Faenza, 1789.

KAUFMAN, T. 1990. Language history in South America. What we know and how to know more, Amazonian linguistics. Studies in lowland Sowth American Languages, ed. by D. L.Payne, Austin: Univ. of Texas Press: 13-74. 
LAFONE QUEVEDO, S. A. (1894a). Los lules, Boletín del Instituto Geográfico Argentino, 15, Buenos Aires: 185-246.

(1894b). Calepino lule-castellano. Vade mecum para el Arte y Vocabulario del Padre Antonio Machoni S. J., Boletín del Instituto Geográfico Argentino, 15, Buenos Aires: 305-85.

(1895). Lengua vilela ó chulupí. Estudio de filología chaco-argentina fundado sobre los trabajos de Hervás, Adelung y Pelleschi, Boletín del Instituto Geográfico Argentino, 16, Buenos Aires: $39-86$ y $87-125$.

LEHMANN-NITSCHE, R. (1924-5). Mitología sudamericana, XI: La astronomía de los vilelas, Revista del Museo de La Plata, 27, serie 3, vol. 4: 210-33.

LOUKOTKA, C. (1968). Classification of South American Indian Languages, Editor J. Wilbert, Los Angeles: Univ. of California, Latin American Center, References Series, vol. 7.

LOZANO, E. (1964). Estudios sobre el vilela, Boletín de Filología, Montevideo, 10 (61-62): 151-7. .(1970a). Textos Vilelas, La Plata: CEILP.

(1970b). La mujer infiel, Anales del.Instituto de Lingüística, 10, Mendoza:. 77-100.

.(1977). Cuentos secretos vilelas: I. La mujer tigre, VICUS, Cuadernos, Lingüística, 1, Amsterdam: 93-116.

LLAMAS, A. de. 1910. Uakambabelté ó Vilela, Lenguas indígenas ab-orígenes. Mi contribución primera al estudio de la historia antigua, Corrientes: Tip. y Enc. de Teodoro Heinecke.

MACHONI, A. 1877. Arte y vocabulario de la Lengua Lule y Tonocoté, Buenos Aires: P. E. Coni. Reproducción de la ed. de Madrid, 1732, con introd. de J. Lársen.

MARTÍNEZ CROVETTO, R. N. (1965). Estudios etnobotánicos. II. Nombres de plantas y su utilidad según los indios vilelas del Chaco, Bonplandia, 2 (1), Corrientes: 1-23.

.(1968). Algunos juegos de los indios vilelas, Etnobiológica, 5 (2), Corrientes: Univ. Nac. del Nordeste, Fac. de Agronomía y Veterinaria: 1-23.

(1995). Zoonimia y etnozoología pilagá, toba, mocoví, mataco y vilela, Buenos Aires: Univ. de Buenos Aires, Fac. de Filosofía y Letras, Inst. de Lingüística.

RONA, J. P. 1964. Nuevos elementos acerca de la lengua charrúa, Publicaciones del departamento de Lingüística, 19, Montevideo: Univ. de la República.

SWADESH, M. 1959. Mapas de Clasificación Lingüística de México y las Américas, México: Univ. Nac. Autónoma de México, Cuadernos del Inst. de Historia, Serie Antropológica, 8.

TERÁN, B. 1995. Figuras del panteón vilela, J. Schobinger (comp.) Humanismo Siglo XX. Estudios dedicados a Juan Adolfo Vázquez, San Juan: Ed. Univ. Nac. de San Juan: 187-95.

TOVAR, A. y C. Larrucea de Tovar. 1984. Catálogo de las lenguas de América del Sur, con clasificaciones, indicaciones tipológicas, bibliografía y mapas. Nueva Ed. Refundida. Madrid: Gredos. 